\title{
CERN LHC sensitivity to the resonance spectrum of a minimal strongly interacting electroweak symmetry breaking sector
}

\author{
A. Dobado, ${ }^{1}$ M. J. Herrero, ${ }^{2}$ J. R. Peláez, ${ }^{1}$ and E. Ruiz Morales $^{2}$ \\ ${ }^{1}$ Departamento de Física Teórica, Universidad Complutense de Madrid, 28040 Madrid, Spain \\ ${ }^{2}$ Departamento de Física Teórica, Universidad Autónoma de Madrid, Cantoblanco, 28049 Madrid, Spain
}

(Received 13 December 1999; published 7 August 2000)

\begin{abstract}
We present a unified analysis of the two main production processes of vector boson pairs at the CERN LHC, $V V$-fusion and $q \bar{q}$ annihilation, in a minimal strongly interacting electroweak symmetry breaking sector. Using a unitarized electroweak chiral Lagrangian formalism and modeling the final $V_{L} V_{L}$ strong rescattering effects by a form factor, we describe $q \bar{q}$ annihilation processes in terms of the two chiral parameters that govern elastic $V_{L} V_{L}$ scattering. Depending on the values of these two chiral parameters, the unitarized amplitudes may present resonant enhancements in different angular momentum-isospin channels. Scanning this two parameter space, we generate the general resonance spectrum of a minimal strongly interacting electroweak symmetry breaking sector and determine the regions that can be probed at the CERN LHC.
\end{abstract}

PACS number(s): 12.60.Fr, 12.39.Fe

\section{INTRODUCTION}

One of the main goals of the CERN Large Hadron Collider (LHC) will be to bring some light on the symmetry breaking sector (SBS) of electroweak (EW) interactions. In spite of the impressive agreement of the present data with the standard model (SM) predictions, the origin of EW symmetry breaking remains unknown to a large extent. From direct searches of the SM Higgs boson [1] we know that it has to be heavier than $101 \mathrm{GeV}$ (95\% C.L.), and the fit to EW data [2] gives a $95 \%$ C.L. upper bound of $230 \mathrm{GeV}$. Concerning alternative SBS scenarios, the EW precision measurements disfavor the most simple technicolor model $[3,4]$, but the data are compatible with a general class of strongly interacting SBS [5]. One of the most characteristic signals of this type of models is the enhanced production of longitudinal vector boson pairs $\left(V_{L} V_{L}\right)$ at high energy colliders [6].

The electroweak chiral Lagrangian (EChL) [7] provides a general way to describe the low energy effects of different strongly interacting SBS models, which are represented by different values of the effective chiral couplings. It is inspired in the chiral Lagrangian description of low energy pion dynamics in QCD $[8,9]$. However, the perturbative predictions made with this effective Lagrangian can only describe accurately EW physics at low energies. The reason is that EW observables are given as a truncated series in powers of the external momenta and, therefore, they will always violate the unitarity bounds if we go to high enough energy. In particular, at LHC, the EChL amplitudes involving longitudinal gauge bosons will violate unitarity for values of the effective couplings in the expected range of $10^{-2}$ to $10^{-3}$. Furthermore, these polynomials in the external momenta will not be able to reproduce the main feature of this type of models, that is, the poles associated to possible new heavy resonances generated by the SBS dynamics.

The perturbative EChL predictions can be extended to high energy using unitarization methods [10]. The unitarized amplitudes for $V_{L} V_{L}$ production processes can also reproduce a resonant behavior depending on the values of the effective couplings. Since the effective couplings appearing in $V V$ fusion are different from those in $q \bar{q}$ annihilation, these two processes will not show in general the same pattern of resonances for an arbitrary choice of the effective couplings. However, given that the SBS interactions are strong, their dominant effects in both processes are due to the same strong $V_{L} V_{L}$ rescattering. Thus, using the unitarity relations between $q \bar{q}$ annihilation and $V_{L} V_{L}$ fusion, we describe in this paper both processes only in terms of the two chiral parameters that govern elastic $V_{L} V_{L}$ scattering.

We start giving in Sec. II a brief overview of the chiral Lagrangian description of EW interactions. We summarize the present experimental bounds on the chiral coefficients and discuss which are the relevant ones for the present study. In Sec. III, we provide a unified unitarized description of the two main $V_{L} V_{L}$ production processes at the LHC, showing the spectrum of resonances expected in different regions of the two parameter space. In Sec. IV we apply these techniques to study the effects of the scalar and vector resonances of the SBS in the production of $Z Z$ and $W Z$ pairs at the LHC. First, we briefly describe our calculation of the signal and the main background reactions. In order to obtain conservative predictions, we will restrict our analysis to the cleanest detection modes with the final $W$ and $Z$ bosons decaying into leptons $(e, \mu)$. Finally, in Sec. IV, we perform a systematic study of the significance of the signals for both vector and scalar resonances, and determine the region of the parameter space where these resonances can be probed at the LHC. We give our conclusions in Sec. V.

\section{CHIRAL LAGRANGIAN DESCRIPTION OF ELECTROWEAK INTERACTIONS}

In this work we study minimal strongly interacting symmetry breaking sectors (MSISBS), in which the global symmetry breaking pattern $S U(2)_{L} \times S U(2)_{R}$ down to the custodial $S U(2)_{\text {c }}$ symmetry is the smallest one ensuring [11] that $\rho \approx 1+\mathcal{O}\left(g^{2}\right)[12]$. The only light modes of the SBS are the three Goldstone bosons (GB) associated to this global 
symmetry breaking. The next physical states from the SBS are expected to be heavy resonances at the $\mathrm{TeV}$ scale, generated by the strong interaction dynamics. Since no additional Higgs field is included in this approach, the symmetry has to be realized nonlinearly, with the three GB, $\omega^{a}(x)$ with $a=1,2,3$, gathered in an $\mathrm{SU}(2)$ matrix

$$
U(x)=\exp \left(\frac{i \omega^{a}(x) \tau^{a}}{v}\right),
$$

where $\tau^{a}$ are the Pauli matrices and $v=246 \mathrm{GeV}$.

The EW interactions at low energies can be well described by the electroweak chiral Lagrangian [7], an effective field theory that couples the three GB to the gauge bosons and fermions in an $S U(2) \times U(1)$ invariant way. This Lagrangian has a set of effective operators of increasing dimension that represent the low energy effects of the underlying symmetry breaking dynamics. The $C$ and $P$ invariant bosonic operators up to dimension 4 are

$$
\begin{aligned}
\mathcal{L}_{\mathrm{EChL}}= & \frac{v^{2}}{4} \operatorname{Tr} D_{\mu} U\left(D^{\mu} U\right)^{\dagger}+a_{0} \frac{g^{\prime 2} v^{2}}{4}\left[\operatorname{Tr}\left(T V_{\mu}\right)\right]^{2}+a_{1} \frac{i g g^{\prime}}{2} B_{\mu \nu} \operatorname{Tr}\left(T \mathcal{W}^{\mu \nu}\right)+a_{2} \frac{i g^{\prime}}{2} B_{\mu \nu} \operatorname{Tr}\left(T\left[V^{\mu}, V^{\nu}\right]\right) \\
& +a_{3} g \operatorname{Tr}\left(\mathcal{W}_{\mu \nu}\left[V^{\mu}, V^{\nu}\right]\right)+a_{4}\left[\operatorname{Tr}\left(V_{\mu} V_{\nu}\right)\right]^{2}+a_{5}\left[\operatorname{Tr}\left(V_{\mu} V^{\mu}\right)\right]^{2}+a_{6} \operatorname{Tr}\left(V_{\mu} V_{\nu}\right) \operatorname{Tr}\left(T V^{\mu}\right) \operatorname{Tr}\left(T V^{\nu}\right)+a_{7} \operatorname{Tr}\left(V_{\mu} V^{\mu}\right) \\
& \times\left[\operatorname{Tr}\left(T V^{\nu}\right)\right]^{2}+a_{8} \frac{g^{2}}{4}\left[\operatorname{Tr}\left(T \mathcal{W}_{\mu \nu}\right)\right]^{2}+a_{9} \frac{g}{2} \operatorname{Tr}\left(T \mathcal{W}_{\mu \nu}\right) \operatorname{Tr}\left(T\left[V^{\mu}, V^{\nu}\right]\right)+a_{10}\left[\operatorname{Tr}\left(T V_{\mu}\right) \operatorname{Tr}\left(T V_{\nu}\right)\right]^{2}+\text { e.o.m. terms } \\
& + \text { standard YM terms }
\end{aligned}
$$

where the "e.o.m" terms refer to other operators that can be removed using the equations of motion, the "standard YM terms" stand for the gauge fixing and Faddeev-Popov terms, and we have defined the following combinations of fields:

$$
T \equiv U \tau^{3} U^{\dagger} ; \quad V_{\mu} \equiv\left(D_{\mu} U\right) U^{\dagger}
$$

and the covariant derivative and field strength tensors are given by

$$
\begin{aligned}
D_{\mu} U & \equiv \partial_{\mu} U-g \mathcal{W}_{\mu} U+g^{\prime} U \mathcal{B}_{\mu}, \\
\mathcal{W}_{\mu} & \equiv \frac{-i}{2} \vec{W}_{\mu} \cdot \vec{\tau}, \quad \mathcal{B}_{\mu} \equiv \frac{-i}{2} B_{\mu} \tau^{3}, \\
\mathcal{W}_{\mu \nu} & \equiv \partial_{\mu} \mathcal{W}_{\nu}-\partial_{\nu} \mathcal{W}_{\mu}-g\left[\mathcal{W}_{\mu}, \mathcal{W}_{\nu}\right], \\
B_{\mu \nu} & \equiv \partial_{\mu} B_{\nu}-\partial_{\nu} B_{\mu} .
\end{aligned}
$$

The first term in Eq. (2) has the form of a gauged non-linear sigma model $(\mathrm{NL} \sigma \mathrm{M})$ and is universal, giving the mass of the $W$ and $Z$ bosons and the "low energy theorems" for longitudinal gauge boson scattering [13]. The other operators have model-dependent effective couplings $a_{i}$ that play a double role. First, six of them (from $a_{0}$ to $a_{5}$ ) are needed as counterterms to cancel the divergences generated in a oneloop calculation with the NL $\sigma \mathrm{M}$ Lagrangian without a Higgs boson. Note that these divergences are universal. Therefore, after renormalization, these effective couplings will have a logarithmic dependence on the scale that is universal $[8,9,7,14]$, and a finite piece that depends on the prescription used to renormalize the $\mathrm{NL} \sigma \mathrm{M}$ divergences. In this work, we will use the modified minimal subtraction $(\overline{\mathrm{MS}})$ renormalization prescription for the effective couplings $a_{i}$. Second, the $a_{i}$ coefficients parametrize the low energy effects of the underlying symmetry breaking dynamics. For some par- ticular models of strong symmetry breaking, the values of these effective couplings can be calculated by integrating out the heavy degrees of freedom of the underlying theory, as it has been done for the SM with a heavy Higgs boson [14]. In addition they have also been calculated in the large $N_{T C}$ limit of technicolor models [15], as well as for chiral models within the resonance saturation hypothesis $[9,16,17]$. In all these cases, and for masses of the heavy resonances in the $\mathrm{TeV}$ range, the typical size of these effective couplings in the $\overline{\mathrm{MS}}$ scheme lies in the range $10^{-2}$ to $10^{-3}$.

The EChL formalism has been applied to constrain the effective couplings from EW low energy data. For instance, the couplings $a_{0}, a_{1}$ and $a_{8}$ contribute to the gauge boson self energies up to order $q^{2}$ [18], and are related to the T, S, and $\mathrm{U}$ parameters $[3,14,19]$ as explained below. A one-loop EChL calculation of the self-energy combinations entering the definition of $\mathrm{S}, \mathrm{T}$ and $\mathrm{U}$ gives

$$
\begin{aligned}
S & =\frac{4 e^{2}}{\alpha}\left[\Pi_{33}^{\prime}(0)-\Pi_{3 Q}^{\prime}(0)\right] \\
& =16 \pi\left[-a_{1}^{\overline{M S}}(\mu)+(\mathrm{NL} \sigma \text { M-loops })(\mu)\right], \\
T & =\frac{e^{2}}{\alpha s^{2} c^{2} m_{Z}^{2}}\left[\Pi_{11}(0)-\Pi_{33}(0)\right] \\
& =\frac{8 \pi}{c^{2}}\left[a_{0}^{\overline{M S}}(\mu)+(\mathrm{NL} \sigma \mathrm{M}-\text { loops })(\mu)\right], \\
U & =4 \frac{e^{2}}{\alpha}\left[\Pi_{11}^{\prime}(0)-\Pi_{33}^{\prime}(0)\right] \\
& =16 \pi\left[a_{8}+(\mathrm{NL} \sigma \mathrm{M} \text {-loops })\right],
\end{aligned}
$$


where we have made explicit the $\mu$-dependence of the effective couplings $a_{0}$ and $a_{1}$, and of the contributions from the $\mathrm{NL} \sigma \mathrm{M}$ loops. Note that, once a renormalization prescription is chosen ( $\overline{\mathrm{MS}}$ in our case), the scale dependence of the $\mathrm{NL} \sigma \mathrm{M}$ loops is canceled by the renormalized couplings, so that these self-energy combinations are scale and renormalization prescription independent. Equations (5) provide the value of the S,T,U self-energy combinations in a given model characterized by the values of the $a_{0}, a_{1}$ and $a_{8}$ couplings and, in particular, using the values calculated in [14], they give the SM predictions for a heavy Higgs boson. Following the original paper by Peskin and Takeuchi [3], we now define $\Delta S, \Delta T, \Delta U$ as the differences between the vacuum polarization effects in an underlying theory of EW symmetry breaking and those in the standard model with a reference value of the Higgs boson mass $m_{H}$. We can then use Eqs. (5) to evaluate both the contributions from the underlying theory and the SM (provided that a relatively high Higgs boson reference mass $m_{H} \gg M_{W}$ is chosen), and obtain

$$
\begin{aligned}
\Delta S & =S\left(a_{i}\right)-S_{\mathrm{SM}}\left(m_{H}\right) \\
& =16 \pi\left[-a_{1}^{\overline{M S}}(\mu)+\frac{1}{12} \frac{5 / 6-\log m_{H}^{2} / \mu^{2}}{16 \pi^{2}}\right], \\
\Delta T & =T\left(a_{i}\right)-T_{\mathrm{SM}}\left(m_{H}\right) \\
& =\frac{8 \pi}{c^{2}}\left[a_{0}^{\overline{M S}}(\mu)-\frac{3}{8} \frac{5 / 6-\log m_{H}^{2} / \mu^{2}}{16 \pi^{2}}\right], \\
\Delta U & =U\left(a_{i}\right)-U_{\mathrm{SM}}\left(m_{H}\right)=16 \pi a_{8} .
\end{aligned}
$$

These expressions relate the measured $\Delta S, \Delta T, \Delta U$ values obtained from a fit of the Z-pole observables to the SM with the reference value $m_{H}$ with the chiral effective couplings of the underlying theory. The latest fit [4] to EW data with $m_{H}=300 \mathrm{GeV}$, gives

$$
\Delta S=-0.26 \pm 14, \quad \Delta T=-0.11 \pm 16, \quad \Delta U=0.26 \pm 24,
$$

which imply the following bounds for the three chiral couplings:

$$
\begin{aligned}
a_{0}^{\overline{M S}}(1 \mathrm{TeV}) & =(4.3 \pm 4.9) \times 10^{-3}, \\
a_{1}^{\overline{M S}}(1 \mathrm{TeV}) & =(6.8 \pm 2.8) \times 10^{-3}, \\
a_{8} & =(4.9 \pm 4.7) \times 10^{-3} .
\end{aligned}
$$

Similar bounds have been obtained in [5], where a higher $m_{H}$ reference value has been used in the fit. These results disfavor the simplest models of strong SBS, like a heavy SM Higgs boson and rescaled-QCD technicolor models. Indeed, it has been shown [3] that models with exact custodial symmetry, a dominance of vector resonances, and whose underlying SBS dynamics satisfies the Weinberg sum rules [20], give a negative contribution to $a_{1}$ (that is, a positive contribution to $\mathrm{S}$ ) that is clearly disfavored by the data. However, the effective couplings in Eq. (8) are perfectly compatible with the general hypothesis of a strong SBS [5], because their values are in the expected range and no fine tuning is needed in order to fit the data. The open question is then whether there is a model of underlying SBS dynamics that can explain these values. In this work, we take a phenomenological approach without making any assumption on the underlying theory, and investigate what can we expect at future colliders if the EChL couplings take natural values in the range $10^{-2}$ to $10^{-3}$.

At the CERN $e^{+} e^{-}$LEP-II and Fermilab Tevatron, three more effective couplings $a_{2}, a_{3}$ and $a_{9}$ come into play, through their contribution to the triple gauge boson vertices. A complete 1-loop EChL calculation [19] and a fit to the data could place constraints on these new couplings, but this analysis has not been done so far. In spite of that, indirect bounds [21,22] of the order of $10^{-1}$ for $a_{2}, a_{3}$ and $a_{9}$ and in the range of $10^{-1}$ to $10^{-2}$ for $a_{4}, a_{5}, a_{6}, a_{7}$ and $a_{10}$ that contribute to the quartic gauge boson vertices, can be obtained from the low energy data through their contribution to anomalous vertices in 1-loop calculations.

To summarize, the EW interactions in a MSISBS can be well described at low energy by the EChL, with a set of effective couplings taking values in the range of $10^{-2}$ to $10^{-3}$. The signals at low energy are expected to be small deviations in the EW observables, of a similar size to the EW radiative corrections.

Concerning the LHC, there are already studies of its sensitivity to the $W$ and $Z$ interactions within the non-resonant EChL approach $[23,24]$. Hence, they are limited to moderate energies, due to the breaking of unitarity already mentioned in the Introduction. There is a general agreement that, although the present bounds could be significantly improved, with these non-resonant studies the LHC would be hardly sensitive to values of the chiral parameters down to the $10^{-3}$ level. Our aim in this work is to extend these studies to include resonances without leaving the $\mathrm{EChL}$ formalism. At the next generation of colliders, we will be probing the $W$ and $Z$ interactions at $\mathrm{TeV}$ energies, where the longitudinal components of the weak bosons behave as their corresponding GB. Since the GB are modes of the SBS, their selfinteractions are strong and it is reasonable to expect that they will dominate the standard EW corrections. This allows us to simplify further the description of the strong SBS effects at high energies.

First, since we are assuming that the SBS interactions preserve the custodial $S U(2)_{L+R}$ symmetry, only those operators that are custodial symmetric (once the gauge interactions are switched off) can be generated by pure strong interaction effects, and they are expected to be the relevant ones at high energy. These are the universal term and the operators corresponding to the $a_{i}$ couplings with $i=3,4,5$. The couplings of the custodial breaking operators should be generated with at least a partial contribution from the $U(1)_{Y}$ gauge interaction or other sources of custodial breaking, that we are assuming to be subleading compared with the strong SBS dynamics.

It is possible to reduce further the number of operators needed to describe the dominant effects of the strong SBS 
interactions at high energy colliders. If the strong SBS interactions dominate the EW physics at high energy, the key reaction is the scattering of longitudinal vector bosons, because it can take place through a pure strong interaction amplitude. Then, if we know the scattering amplitudes of longitudinal vector bosons in all the relevant channels, this characterizes the main effects of the strong dynamics. In particular, the main corrections to the EW production of $V_{L} V_{L}$ pairs will be due to their strong rescattering effects [25], and if inelastic channels are neglected, we can parametrize all the electroweak $V_{L} V_{L}$ production mechanisms in terms of only two effective couplings $\left(a_{4}, a_{5}\right)$ that govern the elastic $V_{L} V_{L}$ amplitudes. We discuss in the next section how to make this parametrization in the unitarized-EChL formalism. Note that for the rest of the paper we will drop the $\overline{\mathrm{MS}}$ superscripts.

\section{UNITARIZATION AND RESONANCES IN THE SBS}

\section{A. Elastic $V_{L} V_{L}$ scattering}

At high energies, the scattering amplitudes of longitudinal gauge bosons can be approximated by the corresponding GB amplitudes using the equivalence theorem (ET) [6]. At first sight, it may seem that the ET is incompatible with the use of the EChL, since the ET is valid only at energies $\sqrt{s} \gg M_{W}$ while the EChL is a low energy effective theory. Nevertheless, it has been shown [26] that there is still a window of applicability for the ET together with EChL, valid at lowest order in the weak couplings, and for small chiral parameters. However, in general, if we want to use the ET at energies larger than, say, $1 \mathrm{TeV}$, it is essential that the theory respects unitarity at high energies. This is an additional reason to use the unitarization methods that we discuss in this section.

The accuracy of the ET approximation was also studied in [27], by comparing the $V_{L} V_{L}$ scattering cross section calculated at tree level with the $\mathcal{L}_{\mathrm{EChL}}$, Eq.(2), with and without the ET. The difference between the EChL cross sections calculated with external $V_{L}$ and those calculated with external GB's was found to be $O(1 \%)$ as soon as $\sqrt{s}>500 \mathrm{GeV}$. If, in addition, as in the present work, the GB cross sections are considered at lowest order on the weak couplings, i.e., $O\left(g^{0}\right)$ and $O\left(g^{\prime 0}\right)$ for this subprocess, the previous difference amounts to $O(10 \%)$ for the resonant channels, which are the relevant ones here.

Customarily, GB elastic scattering is described in terms of partial wave amplitudes of definite angular momentum, $J$, and weak isospin, $I$, associated to the custodial $S U(2)_{L+R}$ group. With the EChL, these partial waves, $t_{I J}$ are obtained as an energy (or external momentum) expansion

$$
t_{I J}(s)=t_{I J}^{(2)}(s)+t_{I J}^{(4)}(s)+O\left(s^{3}\right),
$$

where the superscript refers to the corresponding power of momenta. The explicit expressions for these GB amplitudes valid up to $O\left(p^{4}\right)$ are given in the Appendix [28]. As long as we are working at lowest order in the weak coupling constants and we are assuming custodial symmetry in the SBS, these amplitudes only depend on the two parameters $a_{4}$ and $a_{5}$.
It is easy to check that the EChL amplitudes given in Eqs. (9) and (A1) do not satisfy the elastic unitarity condition

$$
\operatorname{Im} t_{I J}(s)=\left|t_{I J}(s)\right|^{2} \Rightarrow \operatorname{Im} \frac{1}{t_{I J}(s)}=-1,
$$

which is simply the partial wave version of the optical theorem. However, they satisfy the following perturbative relation

$$
\operatorname{Im} t_{I J}^{(4)}(s)=\left|t_{I J}^{(2)}(s)\right|^{2}
$$

Whereas this condition is approximately equivalent to the exact one for the relevant energies at LEP, SLC and Tevatron, that is definitely not the case in the $\mathrm{TeV}$ energy region. In general, and for $\left(a_{4}, a_{5}\right)$ parameters of a natural size, $10^{-2}$ to $10^{-3}$, the unitarity violations cannot be ignored at energies beyond $1 \mathrm{TeV}$.

To solve this problem, we are going to unitarize the above amplitudes by means of the inverse amplitude method (IAM) [29-31]. This method has given remarkable results describing meson dynamics further beyond the perturbative regime, and reproducing the first resonances in each $I, J$ channel up to $1.2 \mathrm{GeV}[31,32]$. A simple way to understand the IAM is to realize that, as indicated in Eq. (10), the imaginary part of the inverse elastic amplitude is known exactly at all energies. As a consequence, any unitary elastic amplitude has to satisfy

$$
\frac{1}{t_{I J}(s)}=\operatorname{Re} \frac{1}{t_{I J}(s)}-i \Rightarrow t_{I J}(s)=\frac{1}{\operatorname{Re} t_{I J}^{-1}(s)-i} .
$$

Hence, we only need the EChL to approximate the real part of the inverse amplitude. Formally:

$$
\operatorname{Re} t_{I J}^{-1}=\left(t_{I J}^{(2)}\right)^{-1}\left[1-\operatorname{Re} t_{I J}^{(4)} / t_{I J}^{(2)}+\cdots\right] .
$$

Then, using Eq. (11) we arrive at the final expression for the unitary amplitudes

$$
t_{I J}(s)=\frac{t_{I J}^{(2)}(s)}{1-t_{I J}^{(4)}(s) / t_{I J}^{(2)}(s)}
$$

which are the $O\left(p^{4}\right)$ IAM partial waves that respect strict elastic unitarity at all energies. Note that the low-energy chiral prediction, Eq. (9), is recovered if we re-expand Eq. (14) in powers of $s$, so that we have not spoiled the good features of the EChL.

Concerning resonances, although in our derivation of Eq. (14) we have used Eq. (10) which only holds for physical values of $s$, the very same unitarized amplitudes can be obtained using dispersion theory [31], thus justifying the extension of Eq. (14) to the complex plane. In particular, it can be shown that Eq. (14) has the proper analytical structure with the right cuts. In addition, for certain values of the chiral coefficients, the partial waves from Eq. (14) can have poles in the second Riemann sheet, which can be interpreted as dynamically generated resonances. Thus within this EChL +IAM formalism one can describe resonances without in- 
creasing the number of parameters and, at the same time, respecting chiral symmetry and unitarity at all energies.

Note, however, that since the IAM at $O\left(p^{4}\right)$ can only generate one pair of conjugated poles in the complex- $s$ plane, we can only reproduce one resonance per channel. Hence, when we identify poles with resonances, we are implicitly assuming that the values of $a_{4}$ and $a_{5}$ describe the GB interactions due to the low energy tail of these resonances. The saturation of the chiral parameters by the lightest resonance multiplets, is usually known as the resonance saturation hypothesis $[9,16]$, and the better known strong scenarios are indeed of this type.

Furthermore, non-resonant channels can also be well reproduced, since in this case, the IAM poles will appear at energies so high that the low energy regions look nonresonant. Although the IAM formula still yields poles, they are beyond the applicability limits, where other effects that we are neglecting here can come into play, and we are not allowed to interpret them as resonances.

\section{B. Unitarization of $q \bar{q} \rightarrow V_{L} V_{L}$.}

In order to study the LHC sensitivity to the different resonant scenarios via $V_{L} V_{L}$ production, it is essential to include the $q \bar{q}$ annihilation process. By means of the ET, this process can be estimated from $q \bar{q} \rightarrow \omega \omega$. Let us recall that the couplings of GB to quarks are proportional to their mass. Therefore, as far as the initial quarks are essentially massless the $q \bar{q} \rightarrow z z$ amplitude is negligible, and will be ignored. In addition, the only relevant contribution to $q \bar{q}^{\prime} \rightarrow w z$ comes from the s-channel, where a quark and an anti-quark annihilate producing an $W$ which gives the $w z$ GB pair. After this initial weak process, we expect that the final state will rescatter strongly. In practice, such a $W \rightarrow w z$ interaction can be described with a vector form factor, $F_{V}(s)$, by replacing $g$ by $g F_{V}(s)$ (similarly to what happens for the pion form factor). Due to gauge invariance, $F_{V}(0)=1$.

The low energy EChL prediction for the form factor is given as a series expansion

$$
F_{V}(s)=1+F_{V}^{(2)}(s)+\cdots .
$$

The explicit EChL expression of $F_{V}^{(2)}(s)$ is given in the Appendix, but at this moment it is important to note that it depends on the chiral parameter $a_{3}$, thus introducing another undetermined constant in the analysis.

Since we are only considering strong rescattering effects, the exact two body unitarity condition for the form factor reads

$$
\operatorname{Im} F_{V}(s)=F_{V}(s) t_{11}^{*}(s) .
$$

Note that, according to our assumption that the strong SBS interaction preserves custodial symmetry, and due to the fact that in the final state there are two bosons, there are only three possible $(I, J)$ elastic scattering channels, namely $(0,0),(1,1)$ and $(2,0)$ (as it happens also in pion scattering in the isospin limit). Consequently, for the vector form factor which has $J=1$, the final state can only rescatter in the $(1,1)$ channel.

As in the case of the GB elastic amplitudes, the form factor in Eq. (15) only satisfies unitarity perturbatively, i.e.

$$
\operatorname{Im} F_{V}^{(2)}(s)=F_{V}^{(0)}(s) t_{11}^{(2)} *(s)=t_{11}^{(2)}(s) .
$$

A way to unitarize the form factor is to realize that the unitarity condition (16) tells us that the vector form factor $F_{V}$ should have the same phase and the same poles that the $t_{11}$ partial wave. Therefore,

$$
\frac{F_{V}(s)}{t_{11}(s)}=\frac{\operatorname{Re} F_{V}(s)}{\operatorname{Re} t_{11}(s)} .
$$

Now, we can get an approximation of the modulus of $F_{V}$ using the EChL expressions for $\operatorname{Re} F_{V} / \operatorname{Re} t_{11}$. Using the unitarized expression for $t_{11}$ from Eq. (14), we ensure that the poles and phase of $F_{V}$ are correct. In summary, we arrive at

$$
F_{V} \simeq \frac{1+\operatorname{Re} F_{V}^{(2)}}{1+\operatorname{Re} t_{11}^{(4)} / t_{11}^{(2)}} \frac{1}{1-t_{11}^{(4)} / t_{11}^{(2)}} .
$$

At leading order in the chiral expansion, the first fraction in Eq. (19) is one, and the next order correction depends on the parameter $a_{3}$ through $\operatorname{Re} F_{V}^{(2)}$ and on $a_{4}, a_{5}$ through the elastic amplitude $t$. In addition, we are going to show next that this fraction can be very well approximated to one if the same vector resonance dominates both $F_{V}$ and $t_{11}$. On the one hand, the vector form factor can be unitarized using only its EChL expansion in Eq. (15), as follows:

$$
F_{V}(s) \simeq \frac{1}{1-F_{V}^{(2)}(s)} .
$$

(This formula has been tested successfully in pion physics; see [29] and [33].) With this equation it is possible to generate a pole associated to a vector resonance while keeping the correct low energy behavior, much as it happened for elastic scattering and Eq. (14).

On the other hand, if such a vector resonance dominates the final rescattering of the form factor, it should also be present in the $(I, J)=(1,1)$ scattering amplitude. That is, both the above equation and Eq. (14) should have a resonance at the same mass with the same width. Thus, together with Eqs. (11) and (17), which relate the imaginary parts of $t_{11}^{(4)}$ and $F_{V}^{(2)}$, we get the following relation for the real parts around the pole position, $M_{V}$ :

$$
\operatorname{Re} F_{V}^{(2)}\left(M_{V}\right)=\operatorname{Re} t_{11}^{(4)}\left(M_{V}\right) / t_{11}^{(2)}\left(M_{V}\right),
$$

which means that the first fraction in Eq. (19) can be set equal to one as a very good approximation, not only at low energies but also at all energies, when the resonance dominates the amplitude.

The use of unitarization methods to derive this result could suggest some arbitrariness. However, the above rela- 
tion can also be recast in terms of chiral parameters, using the formulas for $t_{11}^{(4)}$ and $F_{V}^{(2)}$ given in the Appendix. In the $\overline{\mathrm{MS}}$ scheme it reads

$$
a_{3}\left(M_{V}\right) \simeq a_{4}\left(M_{V}\right)-2 a_{5}\left(M_{V}\right)-\frac{1}{12} \frac{1}{16 \pi^{2}},
$$

which, apart from the small last factor, is satisfied in $S U(N)$ models at leading order in the large $N$ expansion. Note that from the strictest point of view of the effective Lagrangian, these three parameters are independent, although once one assumes a particular underlying theory or vector dominance, one could get a relation among them. Indeed, for general models of vector dominance, it is possible to estimate the values of the chiral parameters $[9,16,17]$ for different resonances in terms of their masses and widths. Indeed we have checked that, for typical vector resonance masses in the 700 to $3000 \mathrm{GeV}$ range, the first fraction in Eq. (19) ranges between 1.2 and 1.3 for $\sqrt{s}>500 \mathrm{GeV}$, although both the numerator and the denominator are much larger than one. These are only estimates, although they suggest that with this approximation we would be underestimating the amplitude and therefore our conclusions about the signal would lie on the conservative side.

Thus, at least for scenarios with a vector dominance, the unitarized vector form factor is well approximated by

$$
F_{V}(s) \simeq \frac{1}{1-t_{11}^{(4)}(s) / t_{11}^{(2)}(s)},
$$

which is completely determined by the unitarized $t_{11}(s)$ amplitude and depends only on $a_{4}$ and $a_{5}$. This approach has also been applied to the pion form factor and it reproduces the $\rho$ correctly [34].

In models where there is not a vector resonance saturating the $I=1, J=1$ channel, we do not expect a significant enhancement of the vector form factor.

\section{Resonances}

The IAM was first applied to the SBS of the EW theory in [10], to study the signals at the LHC of several specific choices of $a_{4}$ and $a_{5}$ that correspond to models with rescaled-QCD or Higgs-like resonances. The complete theoretical study of the resonances that are generated in the $\left(a_{4}, a_{5}\right)$-plane was performed in [35]. Since we will use this information in the next section, we review here the basic results.

Scanning the $\left(a_{4}, a_{5}\right)$ parameter space in the range between $10^{-2}$ and $10^{-3}$, we can reproduce the scattering amplitudes for $V_{L} V_{L}$ production in the MSISBS. Furthermore, the position of the poles in these amplitudes will give us the masses and widths of the resonances (see the Appendix for the explicit expressions). We show in Fig. 1 a map of the vector resonances $(J=I=1$ channel $)$ in the $\left(a_{4}, a_{5}\right)$ parameter space. Within our approximations, this partial wave only depends on the combination $a_{4}-2 a_{5}$, so that the straight lines with constant $a_{4}-2 a_{5}$ have vector resonances with

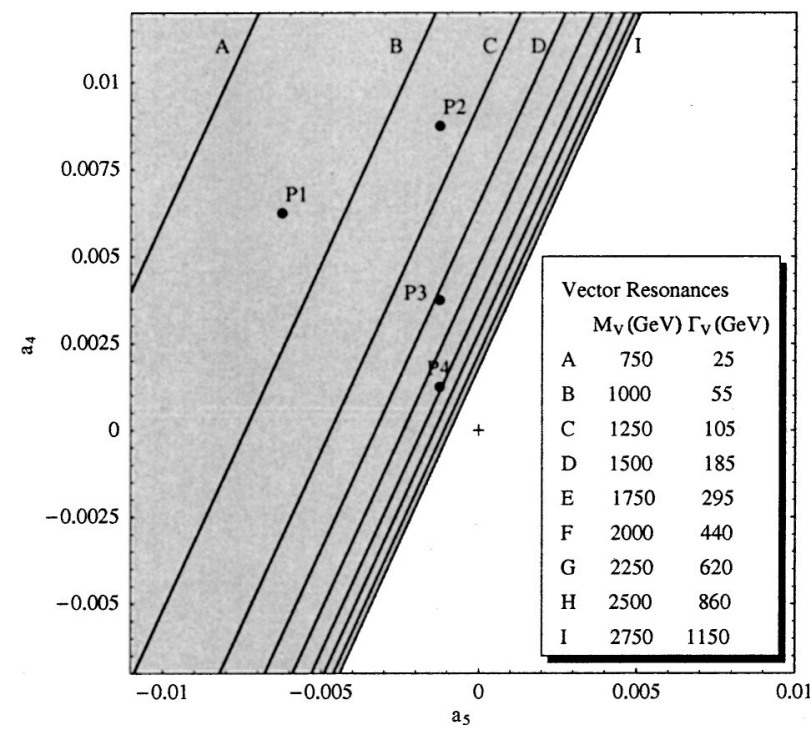

FIG. 1. Vector resonances in the $\left(a_{4}, a_{5}\right)$ parameter space. The chiral couplings are given in the $\overline{\mathrm{MS}}$-scheme at the scale of $1 \mathrm{TeV}$. The $J=I=1$ partial wave only depends on $a_{4}-2 a_{5}$, so that the straight lines have the same physics in this channel. In the table we give the resonance parameters for several lines. The points P1 to P5 will be used as reference models in Sec. IV.

roughly the same mass and width. We give several examples in the table within the figure. In addition we locate five points that we will use later as illustrative examples. The area in blank stands for the case when no resonances or saturation of unitarity is reached below $4 \pi v \simeq 3 \mathrm{TeV}$, which, on general grounds, we expect to be the applicability region of our approach. Similarly, we show in Fig. 2 the map

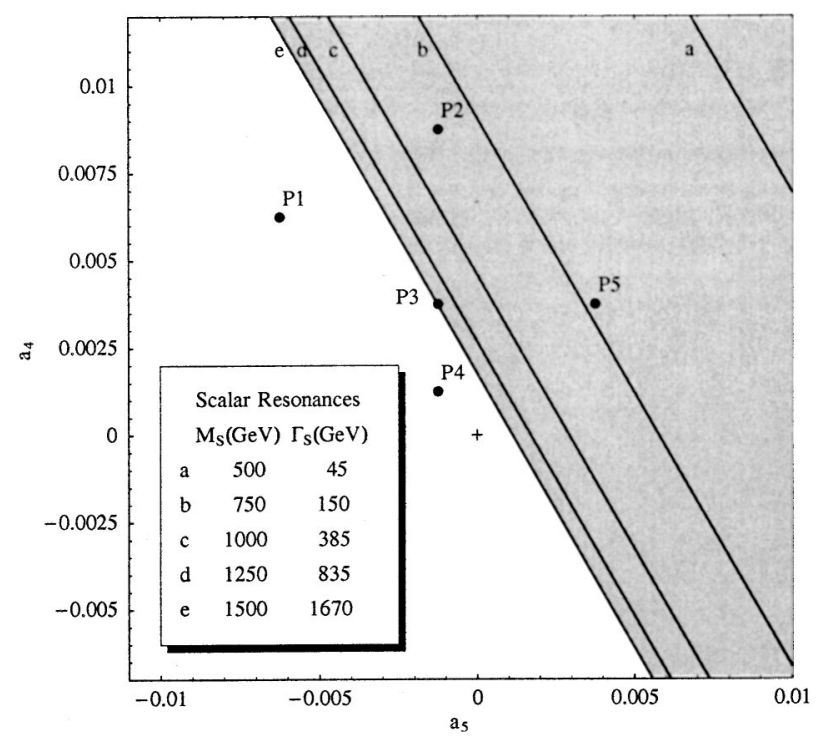

FIG. 2. Scalar neutral resonances in the $\left(a_{4}, a_{5}\right)$ parameter space. The chiral couplings are given in the $\overline{\mathrm{MS}}$-scheme at the scale of $1 \mathrm{TeV}$. The $J=I=0$ partial wave only depends on $7 a_{4}+11 a_{5}$, so that the straight lines have the same physics in this channel. In the table we give the resonance parameters for several lines. The points P1 to P5 will be used as reference models in Sec. IV. 


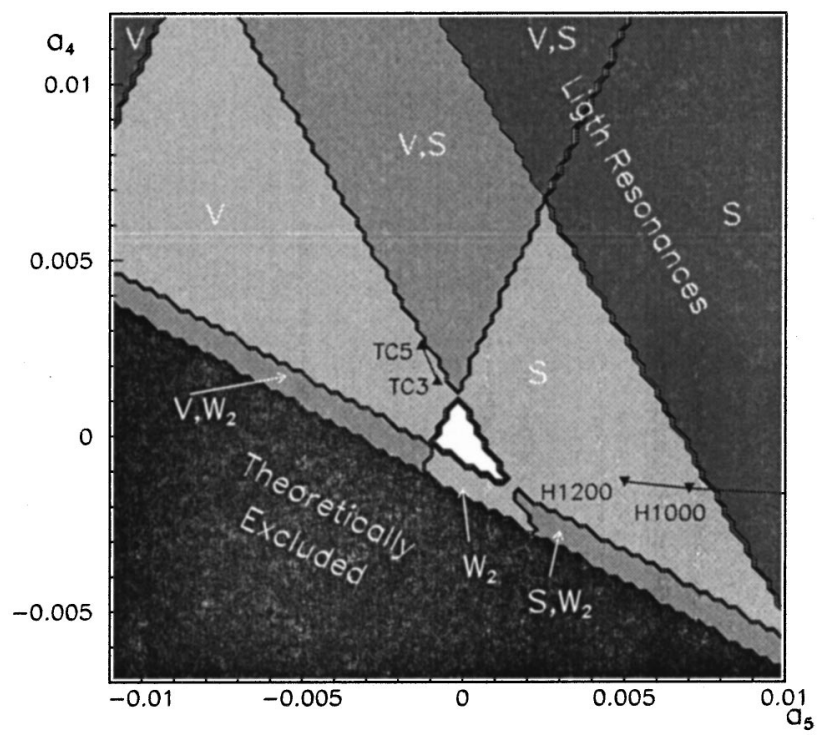

FIG. 3. The general resonance spectrum of a MSISBS in the $\left(a_{4}, a_{5}\right)$ space. The chiral couplings are given in the $\overline{\mathrm{MS}}$-scheme at the scale of $1 \mathrm{TeV}$. V stands for vector resonances, $\mathrm{S}$ for neutral scalar resonances and $W_{2}$ for wide structures that saturate the doubly charged $(I=2)$ channel. For illustration, we have also located the most familiar models of the SM Higgs boson and technicolor, as explained in the text.

of neutral scalar resonances that appear in the $J=I=0$ channel, which only depends on $7 a_{4}+11 a_{5}{ }^{1}$ Incidentally, the fact that the IAM amplitudes only depend on one combination of chiral parameters implies that their mass and width are related by the well known KSFR relation [36].

We do not give the $I=2, J=0$ channel since we do not expect here any resonance in a MSISBS. Intuitively this can be understood from the fact that, at low energies, the $I$ $=2, J=0$ channel is repulsive and therefore we do not expect doubly charged heavy resonances. Furthermore, since we cannot make the slope of a phase shift too negative due to causality (we cannot make an interaction so repulsive that the scattered waves leave the interaction point before they arrive), certain combinations of chiral parameters are excluded theoretically [35]. Taking all this into account, in the $I=2, \quad J=0$ channel we either find a non-resonant behavior or an smooth and wide saturation of unitarity.

We have gathered the information on all these channels in Fig. 3, which is a map of the general resonance spectrum of a MSISBS [35]. Note that depending on the parameters, we can find one scalar resonance $(\mathrm{S})$, one vector resonance $(\mathrm{V})$, two resonances $(\mathrm{S}, \mathrm{V})$, a resonance and a doubly charged wide saturation effect $\left(W_{2}\right)$ or even no resonances below 3 $\mathrm{TeV}$ (white area). For illustrative purposes, we have included the points that correspond to some simple and familiar scenarios: minimal one-doublet technicolor models with 3 and 5 technicolors (TC3 and TC5), and the heavy Higgs boson SM

${ }^{1}$ J.R.P. thanks J.A. Oller for pointing out a mistake in the combination given in [35]. The figures obtained in that paper are nevertheless correct. case, with a tree level Higgs boson mass of 1000 and 1200 $\mathrm{GeV}$ (H1000 and H1200). The black region is excluded by causality constraints on the $I=2, J=0$ wave.

Note that the chiral couplings $a_{4}$ and $a_{5}$ do have a scale dependence $[9,7,14]$

$$
\begin{aligned}
& a_{4}(\mu)=a_{4}\left(\mu^{\prime}\right)-\frac{1}{16 \pi^{2}} \frac{1}{12} \log \frac{\mu^{2}}{\mu^{\prime 2}}, \\
& a_{5}(\mu)=a_{5}\left(\mu^{\prime}\right)-\frac{1}{16 \pi^{2}} \frac{1}{24} \log \frac{\mu^{2}}{\mu^{\prime 2}} .
\end{aligned}
$$

In Figs. $(1,2,3)$ they are given at the scale of $1 \mathrm{TeV}$. Of course, the physical properties of resonances do not change if we change the scale, but their location in the $\left(a_{4}, a_{5}\right)$ plane will be shifted according to the logarithmic running of the effective couplings given in Eq. (24).

Concerning how reliable these predictions are, we should remember that we are neglecting higher order effects on the weak couplings, gauge boson masses and other inelastic channels that could open before $3 \mathrm{TeV}$. We can only make a rough estimate of the accuracy of our predictions based in chiral perturbation theory and meson dynamics or using specific models. From meson-meson scattering, we know that it is possible to reconstruct the lightest resonances from the chiral parameters measured at low energy to within 10 to $20 \%$ of their actual values. We also know that inelastic effects due to states of more than two GB are highly suppressed up to the chiral scale (around $3 \mathrm{TeV}$ in our case). Concerning specific models, we know that we can mimic a heavy Higgs boson scenario or a technicolor scenario within the same range of accuracy. It is worth noting that we expect the predictions to get worse if the resulting resonances become too light. For instance, it is possible to see that the IAM results deviate by more than $20 \%$ from those of the N/D unitarization of a heavy Higgs boson SM if the mass is less than, roughly, $700 \mathrm{GeV}$ [37]. For higher masses the agreement is much better. That is why we have darkened the area where "light resonances" (lighter than $700 \mathrm{GeV}$ ) appear. The results in this area should be interpreted very cautiously. Outside this area we estimate that the predictions of Fig. 3 are reliable within, roughly, a $20 \%$.

\section{GAUGE BOSON PAIR PRODUCTION AT THE CERN LHC}

\section{A. Signal and background processes}

The cleanest way to detect $V V$ pairs at hadron colliders is through the isolated, high- $p_{T}$ leptons produced in their leptonic decay modes. For this reason, we will restrict our analysis to $Z Z$ and $W Z$ production, assuming that their goldplated decay modes $Z Z \rightarrow 4 l$ and $W Z \rightarrow l \nu l l$ (with $l=e, \mu$ ), can be identified and reconstructed with $100 \%$ efficiency. Realistic simulation studies [38] have shown that the inclusion of silver-plated $W^{+} W, Z Z$ and $W^{ \pm} Z$ events, in which one of the gauge bosons decays to jets, can improve the observability of very heavy scalar and vector resonances respectively. However, the study of these channels would re- 
quire a detailed study of QCD backgrounds and jet reconstruction which is beyond the scope of this analysis. Therefore our results, based on gold-plated events, are rather conservative. In addition, since our theoretical scenario does not predict any resonances in the $I=2$ channel, we have not studied like-sign $W^{ \pm} W^{ \pm}$pair production. Nevertheless, this final state could be particularly interesting to test nonresonant models [39,40], due to its small backgrounds.

At LHC, the main production mechanisms of $Z Z$ and $W^{ \pm} Z$ pairs are quark-antiquark annihilation and $V V$ fusion processes. As we explain below, the contribution from nonfusion diagrams with bremsstrahlung of the $V$ bosons is expected to be small after kinematical cuts, and have not been included in our calculation. To evaluate $V V$ fusion processes, we use the effective-W approximation (EWA) [41] and take the gauge bosons as real with leading-order (LO) energy distribution functions. It has been shown by [42] that the LO distributions overestimate the flux of transverse bosons. Since our signal comes from processes involving longitudinally polarized bosons, the uncertainty in the fluxes of transverse $V$ bosons will only affect the backgrounds from SM $V V$-fusion processes, which are probably overestimated. For the parton distribution functions, we have used the CTEQ4 set [43] in all the calculations, evaluated at $Q^{2}=M_{W}^{2}$ in $V V$ fusion processes and at $Q^{2}=s$ in $q \bar{q}$ annihilation and $g g$ fusion processes, with $\sqrt{s}$ being the total center of mass energy of the parton-parton system.

Since we have not included explicitly the decays of the final $W$ and $Z$ bosons to leptons in our programs, we have used the gauge boson variables to set event selection cuts. A first event selection criteria to enhance the strong $V_{L} V_{L}$ production signal over the background is to require high invariant mass $V V$ pairs with small rapidities. We have applied the following set of minimal cuts:

$$
\begin{aligned}
& 500 \mathrm{GeV} \leqslant M_{V_{1} V_{2}} \leqslant 10 \mathrm{TeV} \\
& \left|y_{\text {lab }}\left(V_{1}\right)\right|,\left|y_{\text {lab }}\left(V_{2}\right)\right| \leqslant 2.5 \\
& p_{T}\left(V_{1}\right), p_{T}\left(V_{2}\right) \geqslant 200 \mathrm{GeV} .
\end{aligned}
$$

Indeed, these cuts are also required by the approximations that we have made in our analysis. Given that $V_{L} V_{L}$ $\rightarrow V_{L} V_{L}$ scattering amplitudes are calculated using the ET, our predictions can only be applied to $V V$ boson pairs with high invariant mass. In addition, bremsstrahlung $V$ bosons in non-fusion diagrams are predominantly produced at small angles, and it is a good approximation to neglect their contribution if one restricts the analysis to $V$ bosons with high $p_{T}$ in the central rapidity region. Finally, the $p_{T}$ cut selects $V$ bosons from the signal because they are produced with high $p_{T}$ from the two body decay of a heavy resonance. However, we should keep in mind that our $p_{T}$ distributions have several sources of uncertainty. In $V V$ fusion processes, we have used the EWA assuming collinear $V$ radiation, thus we have neglected the $p_{T}$ of the incident $V$ bosons. In $q \bar{q}$ annihilation processes, we have not included the NLO QCD corrections [44], which are known to increase significantly the distribu- tions at high $p_{T}$ values. In the next section, this minimal set of cuts will be complemented with a more restrictive cut in the invariant mass around the resonances, in order to improve the statistical significance of the signal.

The strong-interaction signal in $Z Z$ production is expected in the fusion channels:

$$
W_{L}^{+} W_{L}^{-} \rightarrow Z_{L} Z_{L}, \quad Z_{L} Z_{L} \rightarrow Z_{L} Z_{L} .
$$

The amplitudes for these processes have been calculated following the approach explained in Sec. III. We have included and estimated the following backgrounds:

$$
\begin{gathered}
q \bar{q} \rightarrow Z Z, \quad 61 \% \\
W^{+} W^{-} \rightarrow Z Z, \quad 18 \% \\
g g \rightarrow Z Z, \quad 21 \%
\end{gathered}
$$

where the percentage is their relative contribution to the total background with the minimal set of cuts. The $Z Z \rightarrow Z Z$ background has not been included since its contribution is known to be negligible compared with $W^{+} W^{-} \rightarrow Z Z$. The continuum from $q \bar{q}$ annihilation has tree level SM formulas. As we have said before, the next to leading order QCD corrections to this process can significantly enhance the tree level cross sections. Therefore, our estimates of the $q \bar{q}$ annihilation background for $Z Z$ production are probably too optimistic. The second background is calculated in the SM at tree level, with at least one transverse weak boson, excluding the Higgs contribution. Finally, the one-loop amplitudes for $g g$ $\rightarrow Z Z$ have been taken from Ref. [45].

For $W^{ \pm} Z$ final states, two processes contribute to the signal:

$$
W_{L}^{ \pm} Z_{L} \rightarrow W_{L}^{ \pm} Z_{L}, \quad q \bar{q}^{\prime} \rightarrow W_{L}^{ \pm} Z_{L}
$$

and the backgrounds included in our analysis are

$$
\begin{gathered}
W^{ \pm} Z \rightarrow W^{ \pm} Z, \quad 18 \% . \\
\gamma W^{ \pm} \rightarrow W^{ \pm} Z, \quad 15 \% . \\
q \bar{q}^{\prime} \rightarrow W^{ \pm} Z, \quad 67 \% .
\end{gathered}
$$

All these backgrounds have SM tree level calculations. The amplitudes for $W^{ \pm} Z \rightarrow W^{ \pm} Z$ have at least one transverse weak boson and exclude the Higgs contribution. In the $q \bar{q}^{\prime}$ $\rightarrow W^{ \pm} Z$ background, we do not include the amplitude with two longitudinal weak bosons, which is considered as part of the signal. The QCD corrections to $q \bar{q}^{\prime}$ annihilation processes would give an enhancement in both the signal and the background, so we expect that they will not modify considerably our estimates of the statistical significance of vector resonance searches. We have not studied the contribution to the background from $t \bar{t}$ production, but it has been shown that it can be efficiently suppressed, after imposing kinematic constraints and isolation cuts to high $p_{T}$ leptons $[38,46]$. 
WZ Final State
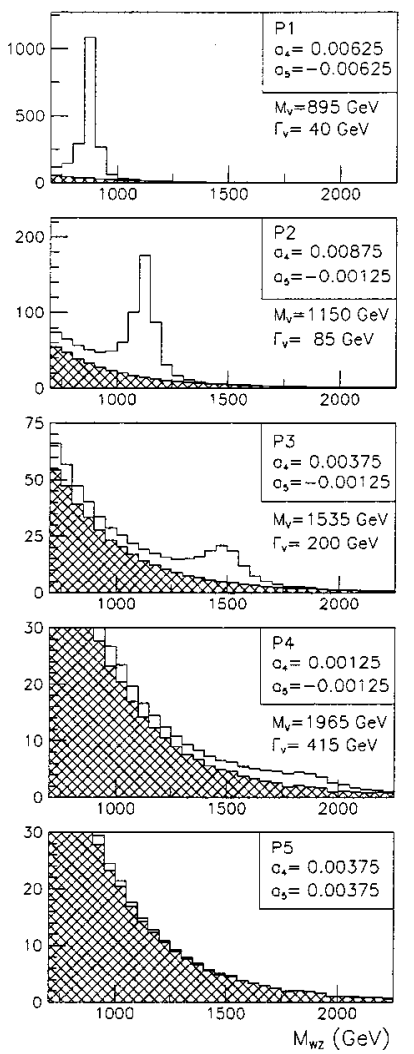

ZZ Find: State
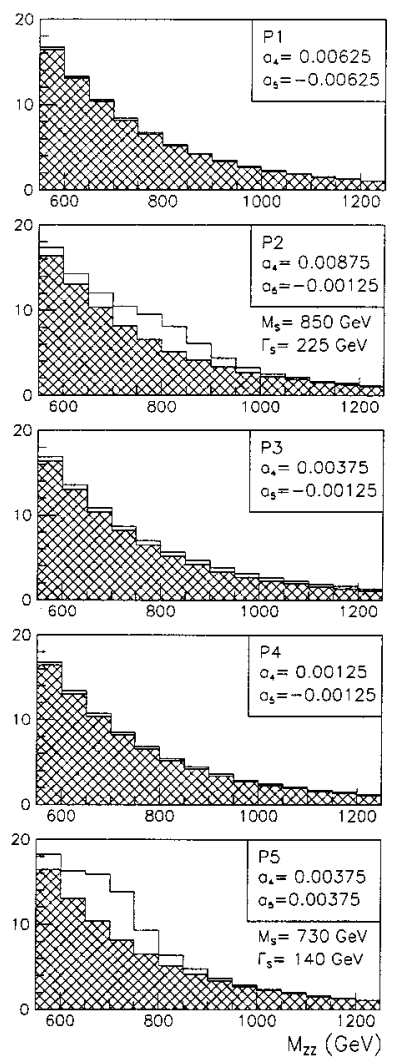

FIG. 4. Distribution of gold-plated $W Z$ and $Z Z$ events for 50 $\mathrm{GeV} M_{V V}$ invariant mass bins, with the minimal set of cuts in Eq. (25). The shaded histogram corresponds to the total background. On top of it we have plotted the signal as a white histogram. We plot (from top to bottom) the predictions for the points P1 to P5 (see Figs. 1 and 2), that represent models with one narrow vector resonance, a vector and a scalar resonance, an intermediate vector resonance, a very wide vector resonance and, finally, a "narrow" scalar resonance.

\section{B. Numerical results}

In order to see the LHC sensitivity to the resonance spectrum described in Sec. III, we have first chosen five representative points in the $\left(a_{4}, a_{5}\right)$ parameter space (see Figs. $1,2)$. Points 1,3 , and 4 represent models containing a $J=I$ $=1$ resonance with masses in the range 900-2000 GeV. Point 5 represents a model with a scalar resonance with mass $730 \mathrm{GeV}$ and a width of $140 \mathrm{GeV}$. Finally, point 2 corresponds to a situation with both a scalar and a vector resonance.

The $M_{V V}$ distributions for these five models are shown in Fig. 4, where we have plotted the signal on top of the background for gold-plated $Z Z$ and $W Z$ events, assuming an integrated luminosity of $100 \mathrm{fb}^{-1}$. The vector resonances in points 1 to 4 can be seen as peaks in the invariant mass distribution for final $W Z$ states. The scalar resonances in points 2 and 5 give small enhancements in the number of $Z Z$ pairs. We can see that, as $a_{4}$ and $a_{5}$ approach the origin, the resonances become heavier and broader, and therefore the signals in the $M_{V V}$ distributions are more difficult to detect. From these plots, it is also evident that it will be much harder to detect scalar than vector resonances. The reasons are: First, that scalars are not significantly produced in $q \bar{q}$ annihilation. Second, the smaller rate of $Z Z$ production from $V V$-fusion. Third, the fact that the branching ratio to leptons is smaller for $Z Z(B R=0.0044)$ than for $W Z$ final states $(B R$ $=0.015$ ), and, finally, that the scalar resonances are approximately six times wider than vector resonances for the same mass.

The relative contribution of the different signal and background processes for $W Z$ and $Z Z$ production at these representative points is given in Tables I and II. In order to enhance the signal to background ratio, we have optimized the cut in $M_{V V}$, keeping events in the region of approximately one resonance width around the resonance mass. The $M_{V V}$ cuts taken in each case are given in the second column of these tables.

TABLE I. Expected number of signal and background gold-plated $W^{ \pm} Z$ events at the CERN LHC with $\mathcal{L}=100 \mathrm{fb}^{-1}$, for four different values of $\left(a_{4}, a_{5}\right)$ that give vector resonances in the $900-2000 \mathrm{GeV}$ mass range. We have applied the cuts in Eq. (25), with the optimized cut in the $V V$-invariant mass indicated in each case. The statistical significance of the signal is given also for an integrated luminosity of $400 \mathrm{fb}^{-1}$.

\begin{tabular}{lccccccccc}
\hline \hline P: $M_{V}, \Gamma_{V}(\mathrm{GeV})$ & Cuts: & Signal & Signal & Signal & Backg. & Backg. & Backg. & $S / \sqrt{B}$ & $S / \sqrt{B}$ \\
$\left(a_{4}, a_{5}\right) \times 10^{3}$ & $\left(M_{V V}^{\min }, M_{V V}^{\max }\right)$ & Fusion & $q \bar{q}$ & Total & Fusion & $q \bar{q}$ & Total & & $\left(400 \mathrm{fb}^{-1}\right)$
\end{tabular}

P1: 894, 39

$(-6.25,6.25)$

$(700,1000)$

$123 \quad 1630 \quad 1743$

74

150

$224 \quad 116$

232

P2: 1150,85

$(-1.25,8.75)$

$(900,1300)$

65

369

434

50

84

134

37

75

P3: 1535,200

$(-1.25,3.75)$

(1250, 1700)

24

56

80

21

27

48

11

23

P4: 1963,416

$(-1.25,1.25)$

$(1500,2350)$

10

12

22

14

16

30

4

8 
TABLE II. Expected number of signal and background gold-plated $Z Z$ events at the CERN LHC with $\mathcal{L}=100 \mathrm{fb}^{-1}$, for two representative values of $\left(a_{4}, a_{5}\right)$ with scalar resonances. We have applied the cuts in Eq. (25) with the optimized cut in the $V V$-invariant mass indicated in each case. The statistical significance of the signal is also given for the cases of ideal forward jet-tagging and for an integrated luminosity of 400 $\mathrm{fb}^{-1}$.

\begin{tabular}{|c|c|c|c|c|c|c|c|c|c|}
\hline $\begin{array}{l}\mathrm{P}: M_{S}, \quad \Gamma_{S}(\mathrm{GeV}) \\
\left(a_{4}, a_{5}\right) \times 10^{3}\end{array}$ & $\begin{array}{c}\text { Cuts: } \\
\left(M_{V V}^{\min }, M_{V V}^{\max }\right)\end{array}$ & $\begin{array}{l}\text { Signal } \\
\text { Fusion }\end{array}$ & $\begin{array}{l}\text { Backg. } \\
\text { Fusion }\end{array}$ & $\begin{array}{l}\text { Backg. } \\
\quad g g\end{array}$ & $\begin{array}{l}\text { Backg. } \\
\qquad \bar{q}\end{array}$ & $\begin{array}{c}\text { Backg. } \\
\text { Total }\end{array}$ & $S / \sqrt{B}$ & $\begin{array}{c}S / \sqrt{B} \\
\text { (jet-tagging) }\end{array}$ & $\begin{array}{c}S / \sqrt{B} \\
\left(400 \mathrm{fb}^{-1}\right)\end{array}$ \\
\hline \multicolumn{10}{|l|}{ P2: 850,225} \\
\hline$(-1.25,8.75)$ & $(600,1050)$ & 15 & 10 & 11 & 34 & 55 & 2 & 5 & 4 \\
\hline $\begin{array}{r}\text { P5: } 750,140 \\
\quad(3.25,3.75)\end{array}$ & $(550,900)$ & 21 & 10 & 14 & 39 & 63 & 3 & 6 & 5 \\
\hline
\end{tabular}

From the results for $W Z$ production, it is clear that the LHC will have an extremely good sensitivity to light vector resonances, due to their production through $q \bar{q}^{\prime}$-annihilation which dominates by far the $V V$-fusion process. As the mass of the vector resonance increases, the $q \bar{q}$ contribution is damped faster than the $V V$ fusion, and both signals become comparable for vector masses around $2 \mathrm{TeV}$. It is also important to note that, in $Z Z$ production, the strong interaction signal appears only in $V V$ fusion diagrams, and therefore to tag forward jets is always convenient in this final state in order to reject non-fusion processes. This is not the case, however, for vector resonance searches in $W Z$ pairs because then the most important contribution comes from $q \bar{q}$ annihilation processes. In these tables, we have also estimated the statistical significance of the signal defined as $S / \sqrt{B}$, assuming integrated luminosities of 100 and $400 \mathrm{fb}^{-1}$. In $Z Z$ final states, we also give the significance of the signal assuming perfect forward jet-tagging.

\section{CONCLUSIONS}

We have presented a unified description of longitudinal gauge boson pair production by fusion and $q \bar{q}$ annihilation just in terms of the $a_{4}$ and $a_{5}$ parameters of the electroweak chiral lagrangian (EChL). Our amplitudes respect unitarity and generate dynamically resonances depending on the values of these parameters. Within this approach, we have studied the sensitivity of the LHC to the general resonance spectrum of the minimal strongly interacting symmetry breaking sector.

From a purely phenomenological EChL approach, and without making any further assumption on the underlying symmetry breaking sector dynamics, the present bounds on the electroweak parameters have room for scenarios where heavy scalar or vector resonances can appear in longitudinal gauge boson pair production processes.

We show in Fig. 5 the regions of the $\left(a_{4}, a_{5}\right)$ parameter space that could be tested at the LHC, giving 3 and 5 sigma contours and assuming integrated luminosities of 100 and $400 \mathrm{fb}^{-1}$.

We can see that there is a central region in the $\left(a_{4}, a_{5}\right)$ parameter space that does not give significant signals in gold-plated $Z Z$ and $W Z$ events. This region corresponds to models in which, either the resonances are too heavy to give a significant enhancement at LHC energies, or there are no resonances in the SBS and the scattering amplitudes are unitarized smoothly. It is a very important issue whether this type of non-resonant $V V$ scattering signals could be probed at the LHC. Some authors [40] have argued that doubly charged $W W$ production could be relevant to test this nonresonant region. But non-resonant $V V$ production distributions would have slight enhancements in the high energy region, and a very accurate knowledge of the backgrounds and the detector performance would be necessary in order to establish the existence of non-resonant signals over the continuum background.

When the sensitivity contours are translated into resonance mass reach limits, our results are in good agreement

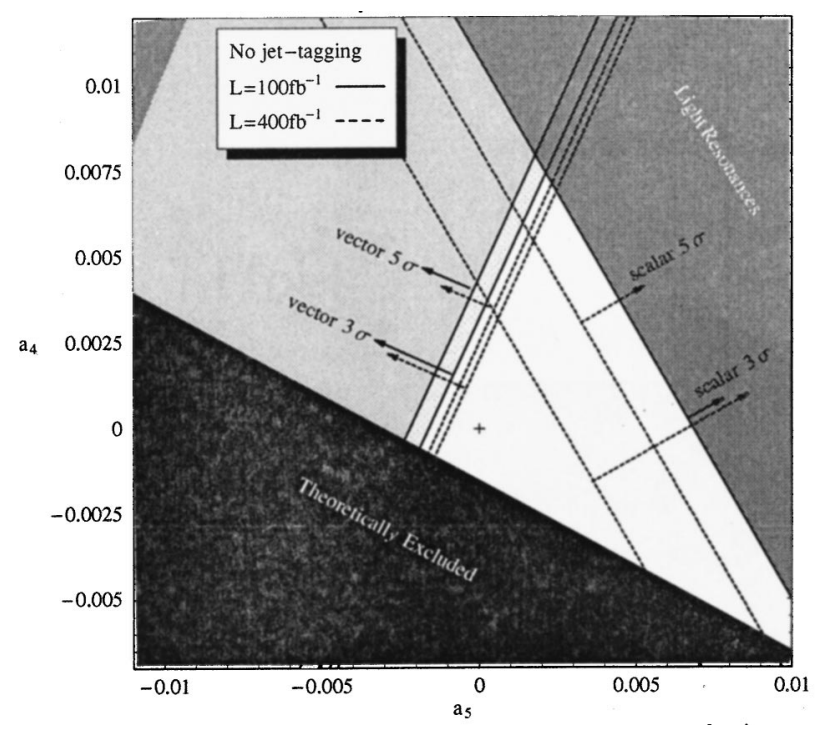

FIG. 5. Sensitivity of the CERN LHC to the resonance spectrum of the strong SBS, with $W Z$ and $Z Z$ gold plated events. In the $\left(a_{4}, a_{5}\right)$ parameter space we represent the $3 \sigma$ and $5 \sigma$ reach with an integrated luminosity of $100 \mathrm{fb}^{-1}$ (solid lines limiting the shaded areas) and $400 \mathrm{fb}^{-1}$ (dashed lines), both for scalar and vector resonances. The chiral couplings are given in the $\overline{\mathrm{MS}}$ scheme at the scale of $1 \mathrm{TeV}$. 
with realistic studies at LHC [38]. We find that, with 100 $\mathrm{fb}^{-1}$, scalar resonances could be discovered $(5 \sigma)$ in goldplated $\mathrm{ZZ}$ events up to a mass of $800 \mathrm{GeV}$ with forward jet-tagging, and vector resonances could be discovered using gold-plated $W Z$ events up to a mass of $1800 \mathrm{GeV}$.

\section{ACKNOWLEDGMENTS}

We acknowledge partial support from the Spanish Ministerio de Educación y Ciencia under CICYT projects AEN971693 and AEN97-1678. E.R.M. also acknowledges support from the Spanish AME Foundation.

\section{APPENDIX}

The EChL predictions $[9,28]$ for the $V_{L} V_{L}$ elastic scattering $t_{I J}$ partial waves, in terms of the $\overline{\mathrm{MS}}$ renormalized $a_{i}(\mu)$ couplings, are

$$
\begin{aligned}
t_{00}^{(2)}= & \frac{s}{16 \pi v^{2}}, \\
t_{00}^{(4)}= & \frac{s^{2}}{64 \pi v^{4}}\left[\frac{16\left(11 a_{5}(\mu)+7 a_{4}(\mu)\right)}{3}\right. \\
& \left.+\frac{1}{16 \pi^{2}}\left(\frac{101-50 \log \left(s / \mu^{2}\right)}{9}+4 i \pi\right)\right], \\
t_{11}^{(2)}= & \frac{s}{96 \pi v^{2}}, \\
t_{11}^{(4)}= & \frac{s^{2}}{96 \pi v^{4}}\left[4\left(a_{4}(\mu)-2 a_{5}(\mu)\right)+\frac{1}{16 \pi^{2}}\left(\frac{1}{9}+\frac{i \pi}{6}\right)\right] \\
t_{20}^{(2)}= & \frac{-s}{32 \pi v^{2}}, \\
t_{20}^{(4)}= & \frac{s^{2}}{64 \pi v^{4}}\left[\frac{32\left(a_{5}(\mu)+2 a_{4}(\mu)\right)}{3}\right. \\
& \left.+\frac{1}{16 \pi^{2}}\left(\frac{273}{54}-\frac{20 \log \left(s / \mu^{2}\right)}{9}+i \pi\right)\right]
\end{aligned}
$$

Note that the projection in angular momentum has been defined as

$$
t_{I J}=\frac{1}{64 \pi} \int_{-1}^{1} d(\cos \theta) P_{J}(\cos \theta) T_{I}(s, t),
$$

where $T_{I}$ is the amplitude in the weak isospin basis.

From these amplitudes, and using Eq. (14), we can obtain the value of the masses and widths of the resonances when they appear. We only have to determine the position of the pole in each channel, and then to identify its real and imaginary parts with the mass and half of the width of the resonance. Thus, for the vector channel, we find

$$
M_{V}^{2}=\frac{v^{2}}{4\left(a_{4}-2 a_{5}\right)+\frac{1}{9(4 \pi)^{2}}}, \quad \Gamma_{V}=\frac{M_{V}^{3}}{96 \pi v^{2}} .
$$

Of course, $M_{V}$ is an observable and cannot depend on the scale. Indeed, the $a_{4}-2 a_{5}$ combination is scale independent [see Eq. (24)]. For the scalar channel we get a trascendental equation

$$
\begin{gathered}
M_{S}^{2}=\frac{12 v^{2}}{16\left(11 a_{5}\left(M_{S}\right)+7 a_{4}\left(M_{S}\right)\right)+101 / 3(4 \pi)^{2}}, \\
\Gamma_{S}=\frac{M_{S}^{3}}{16 \pi v^{2}} .
\end{gathered}
$$

Note that the scale $\mu$ is taken at $M_{S}$. From the above equations it is easy to see that, for equal masses, scalar resonances would be six times wider than vector resonances.

Finally, we give the expression of the $W \rightarrow w z$ vector form factor up to next to leading order in the EChL:

$$
\begin{aligned}
F_{V} & =1+F_{V}^{(2)}(s) \ldots \\
F_{V}^{(2)}(s) & =\frac{s}{(4 \pi v)^{2}}\left[64 \pi^{2} a_{3}(\mu)-\frac{1}{6} \log \frac{s}{\mu^{2}}+\frac{4}{9}+i \frac{\pi}{6}\right]
\end{aligned}
$$

where the $a_{3}(\mu)$ is also given in the $\overline{\mathrm{MS}}$ renormalization scheme. The above equation agrees with the result in [47], where a different renormalization scheme was used.
[1] L3 Collaboration, presentation at LEPC, CERN, 1999, http:// 13www.cern.ch/conferences/talks99.html.

[2] M. Swartz, presentation at the Lepton-Photom Symposium.

[3] M.E. Peskin and T. Takeuchi, Phys. Rev. Lett. 65, 964 (1990); Phys. Rev. D 46, 381 (1992).

[4] Particle Data Group, C. Caso et al., Eur. Phys. J. C 3, 1 (1998).

[5] J.A. Bagger, A.F. Falk, and M. Swartz, Phys. Rev. Lett. 84, 1385 (2000).

[6] J.M. Cornwall, D.N. Levin, and G. Tiktopoulos, Phys. Rev. D
10, 1145 (1974); B.W. Lee, C. Quigg, and H. Thacker, ibid. 16, 1519 (1977); M.S. Chanowitz and M.K. Gaillard, Nucl. Phys. B261, 379 (1985).

[7] T. Appelquist and C. Bernard, Phys. Rev. D 22, 200 (1980); A. Longhitano, ibid. 22, 1166 (1980); Nucl. Phys. B188, 118 (1981).

[8] S. Weinberg, Physica A 96, 327 (1979).

[9] J. Gasser and H. Leutwyler, Ann. Phys. (N.Y.) 158, 142 (1984).

[10] A. Dobado, M.J. Herrero, and T.N. Truong, Phys. Lett. B 235, 
129 (1990); A. Dobado, M.J. Herrero, and J. Terrón, Z. Phys. C 50, 205 (1991); 50, 465 (1991).

[11] P. Sikivie, L. Susskind, M. Voloshin, and V. Zakharov, Nucl. Phys. B173, 189 (1980).

[12] M. Veltman, Acta Phys. Pol. B 8, 475 (1977); Nucl. Phys. B123, 89 (1977).

[13] M. Chanowitz, M. Golden, and H. Georgi, Phys. Rev. Lett. 57, 2344 (1986); Phys. Rev. D 36, 1490 (1987).

[14] M.J. Herrero and E. Ruiz Morales, Nucl. Phys. B418, 431 (1994); B437, 319 (1995); D. Espriu and J. Matias, Phys. Lett. B 341, 332 (1995); S. Dittmaier and C. Grosse-Knetter, Nucl. Phys. B459, 497 (1996); A. Nyffeler and A. Schenk, Phys. Rev. D 53, 1494 (1996); A. Nyffeler, hep-ph/9907294.

[15] T. Appelquist and G.-H. Wu, Phys. Rev. D 48, 3235 (1993).

[16] G. Ecker, J. Gasser, H. Leutwyler, A. Pich, and E. de Rafael, Phys. Lett. B 223, 425 (1989); G. Ecker, J. Gasser, A. Pich, and E. de Rafael, Nucl. Phys. B321, 311 (1989).

[17] J.F. Donoghue, C. Ramirez, and G. Valencia, Phys. Rev. D 39, 1947 (1989).

[18] A. Dobado, D. Espriu, and M.J. Herrero, Phys. Lett. B 255, 405 (1991).

[19] D. Espriu and M.J. Herrero, Nucl. Phys. B373, 117 (1992).

[20] S. Weinberg, Phys. Rev. Lett. 18, 507 (1967).

[21] S. Alam, S. Dawson, and R. Szalapski, Phys. Rev. D 57, 1577 (1998); J.J. van der Bij and Boris Kastening, ibid. 60, 095003 (1999).

[22] O.J.P. Eboli, M.C. Gonzalez-Garcia, and S.F. Novaes, Phys. Lett. B 339, 119 (1994); P. Hernandez and J. Vegas, ibid. 307, 116 (1993).

[23] J. Bagger, S. Dawson, and G. Valencia, Nucl. Phys. B399, 364 (1993); A. Dobado and M.T. Urdiales, Z. Phys. C 71, 659 (1996); A. Dobado, M.J. Herrero, J.R. Peláez, E. Ruiz Morales, and M.T. Urdiales, Phys. Lett. B 352, 400 (1995); H.-J. He, Y.-P. Kuang, and C.P. Yuan, Mod. Phys. Lett. A 11, 3061 (1996); Phys. Rev. D 55, 3038 (1997).

[24] A.S. Belyaev, O.J.P. Eboli, M.C. Gonzalez-Garcia, J.K. Mizukoshi, S.F. Novaes, and I. Zacharov, Phys. Rev. D 59, 015022 (1999).

[25] See, for instance, M. Peskin, SLAC-PUB-5798 (1992).

[26] H.J. He, Y.P. Kuang, and X.Y. Li, Phys. Lett. B 329, 278 (1994); A. Dobado and J.R. Peláez, ibid. 329, 469 (1994);
Nucl. Phys. B425, 110 (1994).

[27] A. Dobado, J.R. Peláez, and M.T. Urdiales, Phys. Rev. D 56, 7133 (1997).

[28] A. Dobado and M.J. Herrero, Phys. Lett. B B228, 425 (1989); 233, 505 (1989); J. Donoghue and C. Ramirez, ibid. 234, 361 (1990).

[29] T.N. Truong, Phys. Rev. Lett. 61, 2526 (1988).

[30] T.N. Truong, Phys. Rev. Lett. 67, 2260 (1991); A. Dobado, M.J. Herrero, and T.N. Truong, Phys. Lett. B 235, 134 (1990).

[31] A. Dobado and J.R. Peláez, Phys. Rev. D 47, 4883 (1993); 56, 3057 (1997).

[32] J.A. Oller, E. Oset, and J.R. Peláez, Phys. Rev. Lett. 80, 3452 (1998); Phys. Rev. D 59, 074001 (1999); 60, 099906 (1999); F. Guerrero and J. A. Oller, Nucl. Phys. B537, 459 (1999).

[33] T. Hannah, Phys. Rev. D 54, 4648 (1996).

[34] A. Dobado, J.R. Peláez, and R. Enriquez-Miranda (in preparation).

[35] J.R. Peláez, Phys. Rev. D 55, 4193 (1997).

[36] K. Kawarabayashi and M. Suzuki, Phys. Lett. 16, 225 (1966); X. Riazuddin and X. Fayyazuddin, Phys. Rev. 147, 1071 (1966).

[37] J.A. Oller, Phys. Lett. B 477, 187 (2000).

[38] ATLAS Technical Proposal, CERN/LHCC/94-43; CMS Technical Proposal, CERN/LHCC/94-38; ATLAS Technical Design Report, Vol.II, CERN/LHCC/99-15.

[39] V. Barger, K. Cheung, T. Han, and R.J.N. Phillips, Phys. Rev. D 42, 3052 (1990).

[40] M. Chanowitz and W. Kilgore, Phys. Lett. B 322, 147 (1994).

[41] S. Dawson, Nucl. Phys. B249, 24 (1985).

[42] J. Lindfors, Z. Phys. C 28, 427 (1985); P.W. Johnson, F.I. Olness, and W.K. Tung, Phys. Rev. D 36, 291 (1987).

[43] CTEQ Collaboration, MSUHEP-60426, CTEQ-604.

[44] J. Ohnemus, Phys. Rev. D 44, 1403 (1991); ibid. 44, 3477 (1991); S. Frixione et al., Nucl. Phys. B383, 3 (1992); S. Frixione, Nucl. Phys. B410, 280 (1993).

[45] E.W.N. Glover and J.J. van der Bij, Nucl. Phys. B321, 561 (1989).

[46] I. Josa, F. Pauss, and T. Rodrigo, in Proc. LHC Workshop, Vol. II, Aachen, 1990, CERN 90-10.

[47] J. Bagger, S. Dawson, and G. Valencia, hep-ph/9204211, Fermilab-PUB-92/75-T. 